\title{
Germanica
}

\section{Zwischen Postdramatik und Postdokumentarismus}

Peter Wagner, Elfriede Jelinek und das Dokumentartheater

Between post-dramatic and post-documentary: Peter Wagner, Elfriede Jelinek

and documentary theater

Entre postdramatique et postdocumentaire. Peter Wagner, Elfriede Jelinek et le théâtre documentaire

\section{Aline Vennemann}

\section{CpenEdition}

\section{Journals}

Édition électronique

URL : http://journals.openedition.org/germanica/2542

DOI : $10.4000 /$ germanica. 2542

ISSN : 2107-0784

\section{Éditeur}

Université de Lille

\section{Édition imprimée}

Date de publication : 30 juin 2014

Pagination : 25-37

ISBN : 9782913857339

ISSN : 0984-2632

\section{Référence électronique}

Aline Vennemann, «Zwischen Postdramatik und Postdokumentarismus », Germanica [Online], 54

2014, Online erschienen am: 30 Juni 2017, abgerufen am 06 Oktober 2020. URL : http://

journals.openedition.org/germanica/2542; DOI : https://doi.org/10.4000/germanica.2542

(C) Tous droits réservés 


\title{
Zwischen Postdramatik und Postdokumentarismus
}

\author{
Peter Wagner, Elfriede Jelinek \\ und das Dokumentartheater
}

\author{
Aline VENNEMANN \\ Université de Haute-Alsace
}

\begin{abstract}
„Kunst, wirkliche, absolute, muß sich jeder Situation gewachsen zeigen und sich an ihr beweisen"1. Mit diesen Worten kommentiert Erwin Piscator in seinem tagebuchartigen Manifest über Das politische Theater (1929) ein einschneidendes Kriegserlebnis: Während der deutschen Frühjahrsoffensive im Jahr 1915 in Flandern gelingt es Piscator nicht, ,, auszuschwärmen“ und sich wie seine Kameraden „einzugraben“. Auf die irritierte Frage des Vorgesetzten nach seiner außermilitärischen Tätigkeit bemerkt der junge Soldat, dass ihm mehr vor seinem Beruf (Schauspieler) als vor den Granaten im Felde graut. Die Absurdität der Situation lässt Piscator nachträglich eine Lektion ziehen: „Kunst als Selbstzweck war nicht mehr imstande, mich zu befriedigen“"2; sie sollte ganz im Gegenteil „,vor der Realität nicht zurückscheuen“. Piscators folgende Theaterarbeit bewahrt nicht nur den Kontakt mit der Wirklichkeit, sondern ersucht ihre Durchdringung. Damit war ein Meilenstein des späteren politischen und dokumentarischen Theaters gelegt, das bis heute neue Formen und Funktionen erprobt ${ }^{3}$.
\end{abstract}

1. - Erwin Piscator, Das politische Theater, Rowohlt, Reinbek/Hamburg, 1963, S. 31.

2. - Ebd., S. 33.

3. - Siehe Birgit Haas, (Hrsg.), Macht: Performativität, Performanz und 
In seiner kritischen Ausrichtung hinterfragt das Dokumentartheater das Verhältnis von Realität, Wunschvorstellung und Fiktion, Wahrheit, Illusion und Lüge. Indem es Dokumenten eine neue Qualität ${ }^{4}$ beimisst, nährt es die zwischen der Authentizität der Quellen und ihrer ästhetischen Übersetzung auf der Bühne oder im Theatertext bestehende Dialektik. Als Kunstform oszilliert es zwischen dokumentarischer Prägnanz und ästhetischer Transgression der wissenschaftlichen Objektivität. Indem es sich dem Erzählen einer Geschichte (Fabel) verweigert, nabelt es sich jedoch auch vom konventionellen, sprich „dramatischen“, Theater ab. Es befindet sich sozusagen in einer Grauzone der traditionellen Dramatik und dessen, was Hans-Thies Lehmann im ausgehenden 20. Jahrhundert als ,postdramatisches Theater" bezeichnet hat ${ }^{5}$.

Seit den 1990er Jahren kann eine Gegentendenz auf den deutschsprachigen Bühnen vermerkt werden 6 . Zunehmend werden neue Formen des szenischen und dramatischen Nach-, Um- und Neuerzählens sowie ihrer Archivierung erfunden. Dabei geht es nicht im dokumentarischen Sinn um eine kompromisslose Darstellung der Wirklichkeit, sondern um die Aufdeckung der faktischen Ambivalenz. Wahrscheinlichkeit als möglicher Schein des Wahren, historische Faktizität, gegenwärtige Nachvollziehbarkeit und dramatische Fiktion stehen auch im Mittelpunkt der Theaterarbeit Peter Wagners. In vielen Punkten treffen die ästhetischen Ansprüche des burgenländischen Künstlers, dessen Werk hier in nuce vorgestellt werden soll, auf Elfriede Jelineks avantgardistische Theaterkunst: Welche Aufbrüche der sogenannten traditionellen Dramatik und der nicht minder umstrittenen Postdramatik vollziehen Jelineks und Wagners Bühnenwerke? Inwiefern handelt es sich um eine Weiterführung bzw. Umkehrung realistischer sowie dramatischer Tendenzen? Sind die Werke der beiden Autoren bezeichnend für das deutschsprachige Gegenwartstheater?

\section{Postdramatik oder Neodramatik? Zum deutschsprachigen Gegenwartstheater}

In der unlängst erschienenen Untersuchung von Schimmelpfennigs dramatischer Raumkonzeption betont Christine Laudahn die erneute

Polittheater seit 1990, Würzburg, Königshausen \& Neumann, 2005, Modern German political drama 1980-2000, Rochester, NY, Camden House, 2003.

4. - Heinar Kipphardt, «Wäre ich Eichmann geworden», Der Spiegel, 15.5.1967.

5. - Hans-Thies Lehmann, Postdramatisches Theater, Verlag der Autoren, Frankfurt/Main, 2005 (1999).

6. - Siehe Birgit Haas, Plädoyer für ein dramatisches Drama, Wien, Passagen Verlag, 2007. In diesem nicht unproblematischen Essay plädiert Haas für eine Aufwertung des dramatischen Textes, der laut Verfasserin in den 1990er Jahren deutlich an Bedeutung gewinnt und weiterhin die Gegenwartsdramatik prägt, z.B. S. 14. 
Hinwendung des zeitgenössischen Theaters zu fiktionalen, mimetischen Formen ${ }^{7}$. Dieser Sachverhalt gilt nicht nur für deutschsprachige Bühnen, sondern auch für das französische Theater, das traditionell stärker an Rhetorik und Rubato gebunden zu sein scheint, wenn auch kaum von einem reinen Texttheater gesprochen werden kann ${ }^{8}$. Patrice Pavis prognostiziert bereits in seiner Theater-Inventur der Jahrhundertwende, dass die „Dramatik in ihrer innovativen Eigenschaft die Inszenierungspraxis hinter sich lassen und den Autoren eine einfache und starke Sprache zurückgeben“, die „,barocke Vorliebe für vollkommene, klassische Texte wieder aufleben“, die „Lust, lebendige Geschichten,Parabeln und Mythen zu erzählen“ wieder aufkommen und die Inszenierung ,keine andere Rolle spielen [wird], als den Sinn des Textes ohne spezielle oder spektakuläre Effekte zu entfalten"9. Dabei wandle sich der Akteur zum alter ego des Rezipienten (oder Autors) und die ,poetische Arbeit an der Sprache“ vertiefe sich. Der „SchauspielerDramatiker“ würde „die Figuren nicht nur verkörpern, sondern sich neben den Text stellen", während das Publikum ihm assistiere. Pavis kommt zu dem Schluss, dass wir dann ,über den Text verfügen und er

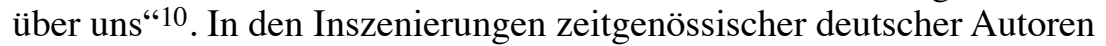
wie Dea Loher oder Marius von Mayenburg behauptet sich der Text tatsächlich als Grundlage. Die Wiederkehr des Dramatischen, welche nicht zuletzt mit der Hinterfragung postmoderner oder postdramatischer Ästhetik einhergeht (wie es Birgit Haas in ihrem „Plädoyer“ exemplarisch zeigt), zeichnet sich parallel zu Theaterformen ab, die das Siegel des Regisseurs oder Autors tragen und eine revidierte Fassung des Regie- und Autorentheaters ${ }^{11}$ darbieten.

Allerdings (und Birgit Haas zum Trotz) widerspricht diese Tatsache nicht Lehmanns Definition des postdramatischen Theaters, wenn er zum Anlass der dritten Auflage seines einschlägigen Werkes schreibt, dass die ,heuristische These“ einer Theaterform, in der sich die Theatermittel „gleichsam aus ihrer jahrhundertealten, mehr oder weniger stringenten

7. - Christina Laudahn, Zwischen Postdramatik und Dramatik: Roland Schimmelpfennings Raumentwürfe, Narr, Tübingen, 2012, S. 11. Die Dissertation wurde an der Ludwig-Maximilian-Universität in München verteidigt.

8. - Siehe dazu Patrice Pavis, «Vorzeitiger Überblick oder vorläufige Schließung wegen Inventur zum Ende des Jahrhunderts», Joachim Fiebach (Hrsg.), Theater der Welt - Theater der Zeit, Arbeitsbuch Theater der Zeit, Berlin 1999, S. 29-35. Pavis betont die Autonomie der Theatertexte (S. 29) und ihre Erschließung bzw. Entfaltung durch die Inszenierung (S. 33-34). Die ,zeitgenössische französische Dramatik“ entspringe daher ,,nicht der Postmoderne und führ[e] nicht zu ihr hin. Sie ha[sse] nichts mehr als den Relativismus und die postmoderne ,Null Bock'-Mentalität" (S. 34).

9. - Ebd., S. 35.

10. - Ebd.

11. - Damit ist nicht das dem Autor « treue », oder gar historisierende Theater gemeint. 
Verlötung“ loslösen, ,keinen theatertheoretischen Universalschlüssel biet[e], sondern bestimmte Zusammenhänge, Verfahren, ästhetische Mittel, Konstellationen, Verknüpfungen wahrnehmbarer mach[e]“. Dabei mag auffallen, ,dass manches, was unerhört neu scheinen kann, nur vergessene oder halbvergessene Potentiale des Theaters wiedererweckt oder an Praktiken der Performance und theatrale Darstellung in anderen Kulturen anknüpft". In diesem Sinn erwähnt Lehmann sogar einen „Fortschritt des postdramatischen Theaters“, der darin bestehe, sich allmählich vom Kunst-Theater zu distanzieren ${ }^{12}$. Ähnlich definieren Patrick Primavesi und Olaf A. Schmitt den von Lehmann geprägten Begriff der Postdramatik ,als Arbeitsformel und nicht etwa als Dogma einer neuen Stilrichtung oder eines isolierten ästhetischen Programms"13.

Die Kategorie des Dramatischen erscheint also nur im Verhältnis zum Postdramatischen einleuchtend und sollte lediglich als Gegenbegriff verwendet werden, nicht aber als Grundbegriff einer „normativen Gattungstheorie“. Nicht nur Lehmann hat unternommen, jenen Aufbruch des klassischen Theaters zu theorisieren. Gerda Poschmann verweist in ihrer Dissertation (1993-1996) auf noch ausstehende Analysekategorien und Beschreibungsformen für die deutsche Gegenwartsdramatik (insbesondere Texte), die als „nicht mehr dramatisch" 14 bezeichnet wird. Poschmann sieht darin einen Gegenentwurf zum aristotelischen Drama, das nicht nur in Freytags legendärer Technik des Dramas (1863), sondern auch in den damaligen Lehrbüchern zur Dramenanalyse modellhaft dargelegt wurde ${ }^{15}$. Da sich die normative Kategorie des Dramatischen für das Theater des ausgehenden 20. Jahrhunderts als nicht mehr tragfähig erwies, beruft sich Poschmann noch vor Erscheinen des Postdramatischen Theaters (1999) auf Andrzej Wirths 1980 erschienenen Essay, in dem die Aufwertung der extraszenischen Achse (Lehmann nennt sie später Theatron-Achse) in jüngeren Theatertexten erstmals besprochen wird ${ }^{16}$. So bezeichnet sie z.B. die Werke Jelineks, Werner Schwabs, Rainald Goetz', Peter

12. - Sämtliche Zitate beziehen sich auf: Lehmann, Ebd., S. Vorwort zur dritten Auflage (o. S.).

13. - Patrick Primavesi, und Olaf A. Schmitt (Hg.), AufBrïche. Theaterarbeit zwischen Text und Situation, Theater der Zeit, Recherchen 20, o.O., 2004, S. 8. Der Sammelband ist Hans-Thies Lehmann zum 60. Geburtstag gewidmet.

14. - Gerda Poschmann, Der nicht mehr dramatische Theatertext: aktuelle Bühnenstücke und ihre dramaturgische Analyse, Tübingen, Niemeyer, 1997.

15. - Poschmann zitiert u.a. Bernhard Asmuth, Einführung in die Dramenanalyse, Stuttgart, 1984 und Heinz Geiger und Hermann Haarmann, Aspekte des Dramas, Opladen, 1987.

16. - Andrzej Wirth, «Vom Dialog zum Diskurs: Versuch einer Synthese der nachbrechtschen Theaterkonzepte», Theater Heute, Heft 1, 1980, S. 16-19. 
Turrinis oder Marlene Streeruwitz' als Unterlaufungen dramatischer Theaterformen.

Das Aufbrechen der dramatischen Form des Theatertextes sowie der Bühnenfassung lässt sich nur notdürftig unter dem Sammelbegriff der Postdramatik subsumieren. Ebenso umstritten scheinen heute die eher veralteten Kategorien des Literatur-, Regie-, Bilder-, Musikoder Tanztheaters ${ }^{17}$. Die intensivere Auseinandersetzung des zeitgenössischen Theaters mit der extraszenischen Wirklichkeit sowie das Effekt-Erheischen gegenwärtiger Inszenierungen lenken verstärkt die Aufmerksamkeit auf sich und mögen als Gegensatz zum dramatischen, repräsentationalen Theater verstanden werden. Handeltes sich hierbeium den Ausbau des Realismus als Weiterführung des Dokumentartheaters der 1970er Jahre oder um eine Aufwertung des Fiktionalismus, der sich wirklichkeitsnaher, dokumentarischer Elemente bedient, um gerade seine künstlerische Freiheit zu unterstreichen?

\section{Peter Wagner und Elfriede Jelinek: Dokumentarische Ambivalenz.}

„Das vorliegende Bühnenstück ist keine Rekonstruktion eines historischen Ereignisses“, heißt es in der Vorbemerkung von Peter Wagners März. Der 24. (1995): „Auch wenn einige der Personen mit Namen versehen sind, deren Träger tatsächlich im Umfeld des Massakers in Rechnitz zu finden waren, läßt dies noch keinen historisch stichhaltigen Rückschluß auf die zur Zeit des Massakers real vorhandenen Schauplätze und Personen zu.“ Diese seien nämlich, so der Autor, „im Sinne eines fiktiven dramatischen Werks frei erfunden und dienen lediglich dem Zweck, eine Parabel auf die Endzeit einer Gesellschaft zu erzählen, wie sie auch auf andere Endzeitalter zutreffen könnte" 18 . Dass jene auktoriale Vorbemerkung nicht als bare Münze zu nehmen, sondern als Erweiterung und Rahmentext der Fiktion zu verstehen ist, haben nicht nur Gérard Genettes Konzeptualisierung des Paratexts gezeigt ${ }^{19}$, sondern auch die Untersuchung des ,pacte de lecture“ durch Philippe Lejeune ${ }^{20}$. Weniger der Textinhalt oder seine Gestaltung bestimmen den fiktionalen Charakter eines Werkes, als textexterne Elemente - sog. „generische Indizien“ wie z.B. Titel, Verleger oder Gattungsbestimmungen auf dem Buchcover.

17. - Primavesi und Schmitt, ebd., S. 8.

18. - Peter Wagner, «März. Der 24», in Tetralogie der Nacktheit, Oberwart, edition lex liszt 12, S. 8-9. 1982.

19. - Gérard Genette, Palimpsestes. La littérature au second degré, Paris, Seuil,

20. - Philippe Lejeune, Le Pacte autobiographique, Paris, Seuil, 1975 ; Je est un autre, Paris, Seuil, 1980. 
Die Vorbemerkung des burgenländischen Autors zum Wirklichkeitsbezug des folgenden Stückes, dessen Titel bereits auf das Massaker von Rechnitz in der Nacht vom 24. auf den 25. März 1945 anspielt, entpuppt sich als zweischneidig. Zum einen bezieht sich der Text auf ein konkretes Endphasenverbrechen im Burgenland: Die Namen der mutmaßlichen Täter und Tatorte werden neben fiktiven Namen zitiert. Der Prolog erinnert knapp an den möglichen Ablauf der Todesnacht und zitiert Zeugen (wie die Repliken im Theatertext) aus Eduard Ernes und Margareta Heinrichs zeitgleichem Dokumentarfilm Totschweigen. A Wall of Silence (1994) ${ }^{21}$. Der Leser erfährt z.B., dass die ,örtliche Parteiprominenz, Gestapo [darunter der Leiter der örtlichen Gestapodienststelle Franz Podezin], Graf und Gräfin [BatthyányThyssen] der Gutsverwalter [Oldenburg], Kellner, HJ-Jungen“ auf dem Gefolgschaftsfest anwesend waren. „Später seien noch die Mädchen aus der Küche zum Tanzen gekommen, berichtet eine Zeugin“, heißt es in indirekter Rede, während ein zweiter Einschub direkt zitiert: „, An Podezin fiel auf, daß er sehr viel tanzte', berichtet ein Kellner"22. Zum anderen betonen die Begriffe „Bühnenstück“, „,fiktiv“, „dramatisches Werk“, „,rei erfunden“, „Parabel“" sowie die Modi des Konjunktiv I und II die hypothetische, fingierte Textnatur. Ihre Präsenz kontrastiert mit den Namen der zeitgenössischen Filmemacher Erne und Heinrich ${ }^{23}$ sowie der lokalen Historikerin Christine Teuschler ${ }^{24}$, auf die sich Wagner explizit beruft.

Offensichtlich spielt hier der Autor mit den Kategorien der Realität und der Fiktion, die er im Sinne Lehmanns zu einem „TheatRealem“ verschmelzen lässt. Kunst hat tatsächlich ,in wechselndem Ausmaß immer außerkünstlerische Beimengungen vom Realen - so wie es umgekehrt ästhetische Faktoren im außerkünstlerischen Bereich gibt (Handwerk)“. Hans-Thies Lehmann erwähnt allerdings in der gleichnamigen Studie, dass ,der ästhetische Prozeß des Theaters nicht im selben Sinn von seiner außerästhetischen realen Materialität abzulösen [sei] wie das ästhetische Ideatum eines literarischen Textes von

21. - Mehr dazu auch in Johanna Jiranek, Darstellungen von Endphasenverbrechen in Literatur und Film aus Österreich aus vergangenheitspolitischer Perspektive, Diplomarbeit, Politikwissenschaft, Universität Wien, Januar 2012 ; «Die Konstruktion des Gedächtnisortes Rechnitz in Literatur, Film und Musik», in ,Die endlose Unschuldigkeit' Elfriede Jelineks Rechnitz,(Der Würgeengel), Pia Janke, Teresa Kovacs, Christian Schenkermayr (Hrsg.), Wien, Praesens Verlag, 2010, p. 329-57.

22. - Wagner, « März. Der 24 », a.a.O., p. 8.

23. - Am 25. Februar 1994 nahm sich Margareta Heinrich das Leben.

24. - Vgl. Georg Gombos, Christiane Gruber, und Christine Teuschler (Hrsg.), „....und da sind sie auf einmal dagewesen: Zur Situation von Flüchtlingen in Österreich. Beispiel Rechnitz", Oberwart, edition lex liszt 12, 1992 ; Eva Schwarzmayer, und Christine Teuschler, Die Mühen der Erinnerung. Zeitgeschichtliche Aufklärung gegen den Gedächtnisschwund, Bd.1, Schulhefte, nr. 105, S. 92-107. 
der Materialität des Papiers und der Druckerschwärze“ 25. Freilich geschieht der „Einbruch des Realen“ innerhalb eines (Theater)Textes indirekter als auf der Bühne, wo der Zuschauer mit konkreten Körpern, sichtbaren Gesten, Formen, Eindrücken und hörbaren Geräuschen konfrontiert wird. Dennoch mag dem entgegnet werden, dass der performative Theatertext - wie ihn auch Wagner und Jelinek konzipieren ebenso „Zeichen eines Zeichens“ (Erika Fischer-Lichte) sein kann und auf den Prozess der Bedeutungskonstituierung verweist. Die gezielten Einschübe von nachprüfbaren Quellen (wie die genau angeführten Aktennamen in der künstlerisch-pädagogisch gestalteten Publikation des Hörspiels Requiem. Den Verschwiegenen aus dem Jahr 200226), paratextuelle Elemente wie Danksagungen oder das oben zitierte Vorwort des Autors lassen sich als ebenso performative und authentische Einschläge des Realen deuten wie materielle Vorbedingungen einer Inszenierung. Die ,im theatralen Schein buchstäblich überspielte“ Wirklichkeit, welche ,jederzeit wieder auftauchen kann“, liegt daher auch dem dramatischen Text zugrunde, den sie jederzeit zu sprengen oder aufzubrechen vermag:

Ohne Reales kein Inszeniertes. Repräsentation und Präsenz, mimetisches Spiel und Performance, Dargestelltes und Darstellungsvorgang: Aus dieser Dopplung hat das Theater der Gegenwart, indem es sie radikal thematisiert und dem Realen Gleichberechtigung mit dem Fiktiven einräumt, ein zentrales Element des neuen theatralen Paradigmas gewonnen, das man postdramatisch nennen kann (und das eine ganze Anzahl anderer Spielarten des Umgangs mit den Theaterzeichen entwickelt hat ${ }^{27}$.

Diese sich auf die Theaterinszenierung beziehende Erkenntnis gilt in gleichem Maße für den geschriebenen Theatertext. Als Zwischenform sei das Programmheft der Inszenierung von März. Der 24. (Regie Walter Davy) am 24. März 1995 in Oberwart (Heimatort Peter Wagners) angeführt, das nicht nur Kommentare zum Massaker und Abbildungen enthält, die die Leichenberge und Gesten damaliger sowie gegenwärtiger Nazianhänger zeigen, sondern auch eine Stichprobe Rechnitzer Erde, die dem Leser-Zuschauer bzw. Zuschauer-Leser konkret mit dem mutmaßlichen Tatort verbindet. Die visuell-materielle Gestaltung des Programms regt unterschiedliche Sinne an und ruft das verdrängte Verbrechen dem lokalen Publikum nicht nur ins Gedächtnis, sondern

25. - Hans-Thies Lehmann, «TheatReales. Notizen», Joachim Fiebach (Hg.), Theater der Welt - Theater der Zeit, Arbeitsbuch Theater der Zeit, Berlin, 1999, S. 65-69, hier S. 65.

26. - Peter Wagner, Requiem. Den Verschwiegenen. Ein Versuch über den Widerstand, Oberwart, edition lex liszt 12, 2002.

27. - Ebd., S. 65-66. 
macht es auch externen Rezipienten zugänglich und in beschränktem Maße erfahrbar. Die Erde erweist sich in der Inszenierung sowie im Text als Leitmotiv und steht allem Anschein nach für die vergrabenen, aufgedeckten und kommenden Verbrechen. Der Programmhefttext entfaltet sich als hybrider Raum, wo sich Zeichenhaftigkeit und Realität überlagern und Abstraktes mit Konkretem innerhalb desselben perzeptiven Deutungsprozesses vereint.

Peter Wagner scheint hier die Realitätsbezogenheit des dokumentarischen Theaters aufzugreifen und gleichzeitig zu veranschaulichen. Das dokumentarische Theater sei, so Peter Weiss in seinen Notizen, ein Theater der Berichterstattung, wo ,Zeugnisse der Gegenwart [...] die Grundlage der Aufführung“ bilden ${ }^{28}$. Doch wenn Weiss zu Beginn der 1970er Jahre noch betont, dass die „kritische Auswahl, und das Prinzip, nach dem die Ausschnitte der Realität montiert werden [...] die Qualität der dokumentarischen Dramatik" 29 ergeben, so geht es Peter Wagner um die kritische Beleuchtung der Dokumente selbst. So können die einmontierten Daten laut der kartonierten Requiem-Edition durchaus „Irrtümer durch zum Teil mangelhaftes und ungenügendes Quellenmaterial“" enthalten. Das Dokumentartheater vor der Jahrtausendwende übte gezielt Kritik an Verschleierung, Wirklichkeitsfälschung und Lügen ${ }^{30}$. Wagner strebt eine differenzierte Offenlegung der Quellenqualität an und stellt die Frage nach ihrer Stichhaltigkeit.

In dem neueren Text Rechnitz (Der Würgeengel) (2009) nimmt Elfriede Jelinek das burgenländische Massaker zum Vorwand einer subtilen Analyse der Historiographie als Konstruktion von Geschichte und der Rolle, die das Theater in geschriebener wie gespielter Form bei der Über- und Vermittlung von Vergangenem einnimmt: „Sie stimmt immer nur ihre Instrumente, die Geschichte, zum Spielen kommt sie aber nur noch selten. Life is very long. Heute allerdings ist so ein Tag, an dem sie mit uns spielt, als würde das Leben überhaupt nicht vergehen“, kommentiert einer der vielen anonymen Boten des Stücks, bevor er seine eigene Funktion folgendermaßen zusammenfasst: „Wir sind ihre Instrumente. Wir stimmen jetzt. Wir stimmen die Geschichte mit uns ab. Unsere Aussagen sollen ja stimmen, und sie sollen übereinstimmen."31 Bewusst greift die Wiener Autorin auf die drei erwähnten Kritikpunkte des Dokumentartheaters zurück und erörtert diese hinsichtlich der offiziellen Geschichtsschreibung. Wie wird Wahrheit bei unzureichenden Quellen ermittelt? Ist Konsens (Übereinstimmung) ein triftiges

28. - Peter Weiss, «Notizen zum dokumentarischen Theater», Rapporte 2, Frankfurt/Main, Suhrkamp, 1971, S. 91.

29. - Ebd., S. 92. [Hervorhebung: A.V.]

30. - Weiss, ebd., S. 92-93.

31. - Elfriede Jelinek, «Rechnitz (Der Würgeengel)», Drei Theaterstücke, Reinbek, Rowohlt, 2009, S. 64. 
Wahrscheinlichkeitsmerkmal? Lässt sich Gewissheit auf ein „Stimmen“ im doppelten Sinne des nominalisierten Verbs stimmen (klangrein machen bzw. sich einigen) und Adjektivs stimmig (passend, richtig) reduzieren? Bezeichnenderweise vermitteln Boten unzusammenhängend die Ereignisse der Rechnitzer Mordnacht. Sie agieren einerseits als unwillige Zeugen, die nichts gesehen und gehört haben wollen, wie auch die Dienerschaft der Gräfin Batthyány-Thyssen, die Eduard Erne und Margareta Heinrich in ihrem Dokumentarfilm interviewt haben ${ }^{32}$, nichts Handfestes berichten; andererseits als Doppelgänger und gemeine Zerrbilder des Historikers, der nichts Selbsterlebtes erzählt, sondern Teile der Vergangenheit anhand von Berichten anderer rekonstruiert und somit kein Bürge der Geschichte an sich, sondern nur des Gesagten sein kann, sprich ein Bote.

Im Unterschied zum Theater ist das Leben weder Thema der Geschichtswissenschaft, noch der Geschichtsschreibung. Das „Spielen“ bzw. das Spiel der Geschichte könne, suggeriert uns der Text, nur auf der lebensechten Bühne des Gesagten stattfinden. Der Schauspieler wird dabei zu ihrem „Instrument“, wie auch der Mensch stets nur ihr bewusster oder unbewusster Träger ist. Theater entpuppt sich demnach als ein Ort, wo Vergangenes erneut eine Rolle spielt, wo Verstummte das Wort ergreifen, wo Vergessenes und Verdrängtes an die Oberfläche treten wie folgender Freudscher Versprecher in Rechnitz andeutet: „Die Personen spielen hier große Oper, nein, Opfer, sie spielen ihre riesigen Rollen, bis ihnen die Rollen zu groß werden und sie sie einfach ablegen" 33 . Während die Historiografie eine „tote" Vergangenheit nachbildet und sich deren Wahrscheinlichkeit einbildet, empfindet das Theater Vergangenes nach und lässt es im performativen Prozess zur Realität werden oder, um mit Lehmann zu sprechen, zur „TheatRealität“.

\section{Offene Theaterformen - eine Zwischenbilanz}

Das Theater von Jelinek und Wagner mag eine Antwort auf die dokumentarischen Tendenzen der 1970er Jahre sein. Schon damals kam man zu der Erkenntnis, dass die unveränderte Übernahme eines authentischen Materials unmöglich sei, da im Kunstprozess stets eine formelle Bearbeitung vollzogen werde: „Die Bühne des dokumentarischen Theaters zeigt nicht mehr augenblickliche Wirklichkeit, sondern das Abbild von einem Stück Wirklichkeit, herausgerissen aus der lebendigen Kontinuität."34 Somit ist auch das Dokumentartheater

32. - Dieser wird sowohl von Peter Wagner als auch von Elfriede Jelinek direkt und indirekt im Theatertext zitiert. Die Interreferenzialität ist besonders auffällig in Rechnitz, so dass man fast von einem medialen Hypotext sprechen könnte.

33. - Jelinek, Rechnitz, S. 80.

34. - Weiss, „Notizen...“ in Rapporte 2, S. 95. 
stets ein ästhetisches Produkt und muss es sogar werden, ,wenn es Berechtigung haben will“"35. Wagner und Jelinek führen Peter Weiss“ Überlegungen weiter, indem sie nicht nur die Objektivität selbst der angegebenen Quellen infrage stellen, sondern auch die vorgegebene Distanz zu den angeführten Belegen. Kann in diesem Sinn von einem post-dokumentarischen Theater die Rede sein, insofern es nicht nur dem Dokumentartheater folgt, sondern dieses zugleich im Hegelschen Sinn aufhebt?

Sowohl für Jelinek als auch für Wagner bilden historische Daten und offizielle Behauptungen die Grundlage der Theatertexte seit den 1990er Jahren. Doch wenden beide Autoren keine strikt dokumentarische Methode an, sondern machen diese selbst zum Thema ihrer Werke. Wagner eröffnet damit dem Leser eine Revision des Dokumentartheaters und „Dokumentarismus“ im Allgemeinen. Im expliziten Anspruch auf Fiktionalität und Wahrhaftigkeit widersprechen seine Werke sogar dem dokumentarischen Grundcharakteristikum der Authentizität. Die postdokumentarische Ästhetik sei daher als eine Gegenbewegung zur Postmoderne zu werten, eruiert Hans-Peter Bayerdörfer ${ }^{36}$. Tatsächlich verschafft die gelegentliche Beziehung auf ein Dokument noch keine dokumentierende Struktur und mag selbst das Gegenteil bewirken. Das Attribut steht vielmehr „,ür die Unverbindlichkeit und Verblasenheit des sogenannten postmodernen Theaters" 37 .

Das Präfix post- hat hierbei eine dreifache Bedeutung: Chronologisch gesehen handelt es sich um ein Theater nach Auschwitz, das wie das Dokumentartheater der unverdauten Missetaten des Holocausts gedenkt. Es spielt also auf das dokumentarische Vermächtnis an. Zugleich weist die Beifügung darauf hin, dass jene Ästhetik überholt ist, ohne sie jedoch deshalb zu annullieren. Postdokumentarisches Theater bezeichnet in diesem Sinn ein Theater der Erinnerung, welches über das Dokument hinaus eine andere, vielleicht höhere oder entferntere Dimension des Wahren bloßlegt, nämlich den Wahrheitsschein der Fiktion bzw. Kunst. Einerseits differenziert die Vorsilbe post- die neue Ausprägung von der vorherigen; andrerseits unterstreicht sie den Konnex der beiden Theaterformen und suggeriert Kontinuität. Schließlich deutet die Partikel auf die Öffnung (oder Brechung) der dokumentarischen Ästhetik. Zum einen verbindet sie die unterschiedlichen Tendenzen des „Postualen“ im ausgehenden 20. Jahrhundert. Zum

35. - Ebd., S. 96.

36. - S. George Taboris post-dokumentarisches Theater und das Bewältigungsproblem auf den deutschen Bühnen (seit 1969), unveröffentlichte Konferenz vom 27. Oktober 1997, Washington University.

37. - Ich beziehe mich auf einen privaten E-Mail-Austausch vom 30. April 2013. 
anderen markiert sie Gegenbewegungen: Postdramatik vs. Postmoderne oder Postdokumentarismus vs. Neodramatik.

Das Theater Elfriede Jelineks und Peter Wagners oszilliert zwischen einer postdramatischen und postdokumentarischen Theaterform, wobei es dokumentarische Taktiken bewusst für dramatische Zwecke einsetzt und simultan dem konventionellen Drama den Rücken kehrt, also postdramatische Züge aufweist und diese auf neodokumentarische Weise dekonstruiert. Dies zeigt z.B. die Schlussszene der März-Inszenierung in Oberwart, die das bewährte dramatische Mittel der Einfühlung für einen dokumentarischen Zweck einsetzt und die Zuschauer das Massaker sozusagen am eigenen Leib erfahren lässt. Im Rechnitz-Text hingegen benutzt Jelinek offensichtlich Quellen, um deren fiktionalen Gehalt freizulegen. Offizielle Diskurse, wissenschaftliche Theorien, Ammenmärchen und Mythen werden konsequent einander gleichgesetzt. Die dadurch entfachten, unerwarteten hermeneutischen Kurzschlüsse extrahieren sowohl Wahres als auch Unwahres. Jelinek beruft sich zweifelsohne auf die dokumentarische Tradition (dies betrifft nicht nur die Stoffauswahl ihrer Stücke, sondern auch das Gewicht des Berichts), um sich anschließend von ihr zu distanzieren und diese zu unterlaufen (durch den Gebrauch von Ironie, Übertreibung oder Wiederholung). Dieses Oszillieren zwischen zwei Authentizitätsbegriffen sowie deren ästhetische Fusion führen zu einer Überwirklichkeit, einem „Hyperrealismus“38, der sich oberhalb und jenseits der Realität befindet und dennoch engen Kontakt zu ihr wahrt. Er bietet ein Konzentrat der Wirklichkeit, das sich im Laufe der Fiktion verdickt. Jelinek versucht dabei, die Wirklichkeit bis zur Kenntlichkeit zu entstellen ${ }^{39}$; Wagner misst dem Dokument eine neue Qualität innerhalb der Kunst bei. Jener Hyperrealismus scheint aus einer besonderen Übersetzungstechnik hervorzugehen. Übersetzen sei nämlich „,wie im Wasser wühlen: was klar erschienen ist wie eine Quelle, ist plötzlich trüb und schlammig“40. Das Theater von Jelinek und Wagner übersetzt in diesem Sinn Realitäten, ohne ihnen den Filter der Glaubwürdigkeit aufzuerlegen. Ganz im Gegenteil: Übertreibung und Entstellung werden Mittel der Offenlegung des eigentlichen Wahrheitsgehaltes.

In diesem Sinn nehmen ihre Werke weniger an der zu Beginn postulierten Rückkehr des Dramatischen teil, als dass sie eine eigene überwirkliche, postdokumentarische Theaterform entwickeln, die abschließend durch den von Marianne Hirsch vor ein paar Jahren eingeführten

38. - Elfriede Jelinek, Christine Lecerf, L'Entretien, Paris, Seuil, 2007, S. 105.

39. - Jacques Lajarrige, «Bis zur Kenntlichkeit entstellen. Effets de déréalisation historique dans In den Alpen et Das Werk», in Françoise Lartillot et Dieter Hornig, Jelinek, une répétition?, Bern, Peter Lang, 2009, S. 5-25.

40. - Elfriede Jelinek, «Für Utta Roy-Seifert», Übersetzergemeinschaft 19811999, Wien, 1999, S. 11. 
Begriff des Nachgedächtnisses (Postmemory) beleuchtet werden kann ${ }^{41}$. Post- bezeichne mehr als einen zeitlichen Abstand oder eine Verortung von Nachwirkungen, sondern übersetze vielmehr, so Hirsch, die Beziehung zwischen direkten Zeugen des kulturellen und kollektiven Holocaust-Traumas und jener Nachgeborenen, die das Trauma lediglich durch Erzählungen, Bilder und Verhaltensweisen erfahren haben ${ }^{42}$. Das „Postgedächtnis“ erinnert nicht wirklich die Vergangenheit, sondern evoziert sie kraft Einbildung, Vorstellung und kreativer Schöpfung 43 . Ähnlich verleiht Elfriede Jelineks und Peter Wagners postdokumentarische Postdramatik dem Medium Theater eine kritisch-mnemonische Funktion, die sie jedoch zugleich unterläuft und infrage stellt.

\section{Bibliografie}

Asmuth, Bernhard, Einfïhrung in die Dramenanalyse, Stuttgart, Metzler, 1984.

Geiger, Heinz und Haarmann, Hermann, Aspekte des Dramas. Eine Einführung in die Theatergeschichte und Dramenanalyse, Opladen, Westdeutscher Verlag, 1987.

Genette, Gérard, Palimpsestes. La littérature au second degré, Paris, Seuil, 1982 / Paratexte. Das Buch vom Beiwerk des Buches, übersetzt von Dieter Hornig, Frankfurt, Campus-Verlag, 1989.

Gombos, Georg, Gruber, Christiane, Teuschler, Christine (Hrsg.), ... und da sind sie auf einmal dagewesen: Zur Situation von Flüchtlingen in Österreich. Beispiel Rechnitz, Oberwart, edition lex liszt 12, 1992.

Haas, Birgit, Modern German political drama 1980-2000, Rochester, NY, Camden House, 2003.

- Plädoyer für ein dramatisches Drama, Wien, Passagen Verlag, 2007.

Haas, Birgit (Hrsg.), Macht: Performativität, Performanz und Polittheater seit 1990, Würzburg, Königshausen \& Neumann, 2005.

Kipphardt, Heinar, «Wäre ich Eichmann geworden », Der Spiegel, 15.5.1967.

Hirsch, Marianne, « The Generation of Postmemory », in Poetics Today 29 : 1, spring, 2008. S. 103-128.

Jelinek, Elfriede, « Rechnitz (Der Würgeengel)», Drei Theaterstücke, Reinbek, Rowohlt, 2009, S. 53-205.

- « Für Utta Roy-Seifert », Übersetzergemeinschaft 1981-1999, Wien, 1999.

Jelinek, Elfriede und Lecerf, Christine, L'Entretien, Paris, Seuil, 2007.

41. - Marianne Hirsch, «The Generation of Postmemory», in Poetics Today 29 : 1 , spring, 2008. S. 103-128.

42. - Ebd., S. 106.

43. - Ebd., S. 107. 
Jiranek, Johanna, Darstellungen von Endphasenverbrechen in Literatur und Film aus Österreich aus vergangenheitspolitischer Perspektive, Diplomarbeit, Politikwissenschaft, Universität Wien, Januar 2012.

- «Die Konstruktion des Gedächtnisortes Rechnitz in Literatur, Film und Musik », in «Die endlose Unschuldigkeit». Elfriede Jelineks Rechnitz (Der Würgeengel), Pia Janke, Teresa Kovacs, Christian Schenkermayr (Hrsg.), Wien, Praesens Verlag, 2010, S. 329-57.

Lajarrige, Jacques, « Bis zur Kenntlichkeit entstellen. Effets de déréalisation historique dans In den Alpen et Das Werk », in Françoise Lartillot et Dieter Hornig, Jelinek, une répétition ?, Bern, Peter Lang, 2009, S. 5-25.

Laudahn, Christina, Zwischen Postdramatik und Dramatik: Roland Schimmelpfennigs Raumentwürfe, Tübingen, Narr, 2012.

Lehmann, Hans-Thies, Postdramatisches Theater, Frankfurt/Main, Verlag der Autoren, 2005.

- « TheatReales. Notizen », Joachim Fiebach (Hrsg.), Theater der Welt - Theater der Zeit, Arbeitsbuch Theater der Zeit, Berlin, 1999, S. 65-69.

Lejeune, Philippe, Le pacte autobiographique, Paris, Seuil, 1975.

- Je est un autre, Paris, Seuil, 1980.

Pavis, Patrice, « Vorzeitiger Überblick oder vorläufige Schließung wegen Inventur zum Ende des Jahrhunderts », Joachim Fiebach (Hrsg.), Theater der Welt - Theater der Zeit, Arbeitsbuch Theater der Zeit, Berlin 1999, S. 29-35.

Piscator, Erwin, Das politische Theater, Reinbek/Hamburg, Rowohlt, 1963.

Poschmann, Gerda, Der nicht mehr dramatische Theatertext: aktuelle Bühnenstücke und ihre dramaturgische Analyse, Tübingen, Niemeyer, 1997.

Primavesi, Patrick und Schmitt, Olaf A. (Hrsg.), AufBrüche. Theaterarbeit zwischen Text und Situation, Theater der Zeit, Recherchen 20, 2004.

Schwarzmayer, Eva und Teuschler, Christine, Die Mühen der Erinnerung. Zeitgeschichtliche Aufklärung gegen den Gedächtnisschwund, Bd.1, Schulhefte, Nr. 105, S. 92-107.

Wagner, Peter, « März. Der 24 », in Tetralogie der Nacktheit, Oberwart, edition lex liszt 12, 1995.

- Requiem. Den Verschwiegenen. Ein Versuch über den Widerstand, Oberwart, edition lex liszt 12, 2002.

Weiss, Peter, « Notizen zum dokumentarischen Theater », Rapporte 2, Frankfurt/ Main, Suhrkamp, 1971, S. 91-104.

Wirth, Andrzej, « Vom Dialog zum Diskurs: Versuch einer Synthese der nachbrechtschen Theaterkonzepte », Theater Heute, Heft 1, 1980, S. 16-19. 
